\title{
AGENCIA DE AUTOCUIDADO EN ALUMNOS DE NUEVO INGRESO EN LA UNIDAD ACADÉMICA MULTIDISCIPLINARIA ZONA MEDIA
}

\section{AGENCY OF SELF CARE IN STUDENTS OF NEW ENTRANCE IN THE UNITY ACADEMIC MULTIDISCIPLINARY ZONA MEDIA}

\author{
Ma. del Rocío Rocha Rodríguez \\ Amaya GonzÁlez Cristela ** \\ EufEMia JuÁrEZ QuiNTERO *** \\ Araceli Olvera Martínez ${ }^{* * *}$ \\ Pinita Agripina Márquez Ponce ${ }^{* * * *}$ \\ Sofía Rosales Guevara ${ }^{* * * * *}$ \\ MinerVa GarCía Rangel ${ }^{* * * * * *}$
}

\begin{abstract}
RESUMEN
Objetivo: Determinar la agencia de autocuidado en los estudiantes de nuevo ingreso en la Unidad Académica Multidisciplinaria Zona Media. Material y método: Estudio descriptivo, correlacional, de corte transversal. Muestra de 281 alumnos de nuevo ingreso de la Unidad Académica Multidisciplinaria Zona Media de México, de las diferentes carreras. Resultados: El cuestionario fue contestado por un total de 315 estudiantes, quedando 34 excluidos, de acuerdo a los criterios de inclusión y exclusión antes mencionados. Por lo tanto, se consiguió un total de participación de 281 alumnos. Encontramos que el 54\% de la población en estudio maneja un nivel de autocuidado medio, el $42 \%$ un alto nivel de autocuidado y solo el 4\% un nivel bajo. Conclusión: El 100\% de los estudiantes conocían el concepto de autocuidado, por lo que se podría deducir que el conocimiento específico acerca de autocuidado no influye en la realización de menores o mayores prácticas de autocuidado; la Universidad en sí no constituyó una gran enseñanza de autocuidado, por lo que en este aspecto le corresponde fortalecer en sus alumnos el conocimiento y las habilidades para que puedan tomar decisiones acerca de su salud, a través de una mejor promoción.
\end{abstract}

Palabras clave: Agencia, autocuidado, estudiantes, enfermería.

\footnotetext{
* Enfermera, Profesora investigadora Unidad Académica Multidisciplinaria Zona Media, Integrante del Cuerpo Académico Cuidado de la Salud. Universidad Autónoma de San Luis Potosí. Email: rocio@uaslp.mx

Estudiante de Licenciatura en Enfermería. Unidad Académica Multidisciplinaria Zona Media. Universidad Autónoma de San Luis Potosí. Email: cristela_023@hotmail.com

${ }^{* * *}$ Estudiante de Licenciatura en Enfermería. Unidad Académica Multidisciplinaria Zona Media. Universidad Autónoma de San Luis Potosí. Email: hannasimpson@hotmail.com

${ }^{* * * *}$ Estudiante de Licenciatura en Enfermería. Unidad Académica Multidisciplinaria Zona Media. Universidad Autónoma de San Luis Potosí. Email: licon_25@hotmail.com

${ }^{* * * * *}$ Estudiante de Licenciatura en Enfermería. Unidad Académica Multidisciplinaria Zona Media. Universidad Autónoma de San Luis Potosí. Email: rocio@uaslp.mx

${ }_{* * * * * *}$ Estudiante de Licenciatura en Enfermería. Unidad Académica Multidisciplinaria Zona Media. Universidad Autónoma de San Luis Potosí. Email: rocio@uaslp.mx

${ }_{* * * * * * *}$ Maestra en Salud Pública. Profesora investigadora Unidad Académica Multidisciplinaria Zona Media, integrante del Cuerpo Académico Cuidado de la Salud. Universidad Autónoma de San Luis Potosí. Email: minerva.garcia@uaslp.mx
} 


\section{ABSTRACT}

Objective: Determine the self-care agency in the freshmen in Multidisciplinary Academic Unit Zona Media. Design: Provide information making them partners for a proper personal care to each of them, counting with the support of teachers and advisers to carry out previous interventions of self-care using didactic and practical material. Methodology: Descriptive, study Correlacional, non-Experimental and transversal cutting. It shows again of 281 student's entrance of the Unidad Académica Multidisciplinaria Zona Media of the different careers. Results: The questionnaire was answered by a total of 315 students, being excluded 34, according to the afore mentioned criteria of inclusion and exclusion. Therefore, it got a total of 281 student participation. We found that $54 \%$ of the population under study manage a level of self-management, $42 \%$ a high level of self-care and only $4 \%$ a low level. Conclusion: $100 \%$ of the students knew the concept of self-care, so it could deduce that specific knowledge about self-care has no influence on the realization of juvenile or older practice of self-care; the University itself, did not constitute a great teaching of self-care, so here is strengthen in students the knowledge and skills so they can make decisions about your health, through a better promotion.

Key words: Agency, self-care, students, nursing.

Fecha recepción: 28/10/13 Fecha aceptación: 06/03/15

\section{INTRODUCCIÓN}

El tener conocimiento de autocuidado hoy en día es un tema de valiosa importancia, la población juvenil tiene características que conllevan a no buscar asesoría en cuestiones de salud, higiene, alimentación, sexualidad, embarazos, abortos, infecciones de transmisión sexual, incapacidades, accidentes de tráfico, muertes, consumo de alcohol y adicciones (1-3). Burmuhl Moore ${ }^{1}$ menciona que el desarrollo de habilidades como: razonar dentro de un sistema de autocuidado, estar motivado para cuidar a uno mismo, tomar decisiones y llevarlas a cabo, emplea un repertorio de aptitudes cognitivas, perceptuales y de comunicación, para llevar acabo un estado de autocuidado favorable para su salud, pero es importante mencionar que el ser humano se caracteriza por realizar pocas acciones de autocuidado a pesar de tener los conocimientos

\footnotetext{
${ }^{1}$ Burmuhl Moore EC. Factores asociados a la agencia de autocuidado de los estudiantes de 3er y 4 to año enfermería, Universidad Austral de Chile (UACH), segundo semestre 2006. [Tesis de licenciatura]. Chile: Universidad Austral de Chile; 2007.
}

para desarrollarlos $(5,6,7)$. El autocuidado en jóvenes es muy relevante ya que es una etapa caracterizada por cambios rápidos en el desarrollo físico, cognitivo y sexual, importante en los procesos de construcción de autoestima, autonomía, formación de la autoimagen y preparación para la adultez. Por lo tanto es importante prestar atención a las prácticas de vida que ejercen los jóvenes, motivados por diversos ejes de interés, entre ellos la familia, la escuela y la sociedad en general $(8,9)$. Existe gran influencia en el ambiente de los centros universitarios sobre el estilo de vida de los estudiantes, los adolescentes pasan de una rutina regular de actividad física a una rutina cargada de inactividad física, esto afecta su estilo de alimentación, debido a la falta de tiempo para preparar alimentos saludables, falta de capacidades y acciones de autocuidado $(10,11,12)$.

El concepto central de la teoría de Orem es el autocuidado, el cual consiste en la práctica de las actividades que las personas maduras, $o$ que están madurando, inician y llevan a cabo en determinados períodos de tiempo, por su propia parte y con el interés de mantener un funcionamiento vivo y sano, y continuar con 
el desarrollo personal y el bienestar mediante la satisfacción de requisitos para las regulaciones funcional y del desarrollo; igualmente, Orem define la actividad de autocuidado como la compleja habilidad adquirida por las personas maduras, o que están madurando, que les permite conocer y cubrir sus necesidades continuas con acciones deliberadas, intencionadas, para regular su propio funcionamiento y desarrollo humano (13).

Además, Orem devela el origen del autocuidado como "un comportamiento aprendido", y las actividades de autocuidado como acciones que se aprenden e internalizan tras la interacción e influencias del medio y la vida cultural del grupo al cual pertenece el individuo. Por lo tanto, las acciones de autocuidado se consideran no innatas, se viven y dependen de las creencias, de la interacción con los diversos factores y estímulos con los que se rodea y con los cuales el individuo experimenta, como costumbres y prácticas habituales de la familia y comunidad de la cual se es parte. Aparece como condicionante de la salud, por lo tanto, el contexto sociocultural, que entrega al individuo los requisitos de autocuidado universales, los cuales son independientes del estado de salud, la edad o el nivel de desarrollo y que se refieren al mantenimiento de un aporte suficiente de aire, agua, alimentos, provisión de cuidados asociados con los procesos de eliminación de excrementos, mantenimiento del equilibrio entre la actividad y el reposo, entre la soledad y la interacción social, prevención de los peligros para la vida, el funcionamiento humano y el bienestar humano, promoción del funcionamiento y desarrollo humano dentro de los grupos sociales de acuerdo con el potencial y talento de las personas $(14,15)$.

Por lo tanto, las instituciones de educación superior tienen la tarea de aportar conductas que nos ayuden a fomentar el autocuidado, dependiendo de las condiciones sociales en las cuales viven y trabajan las personas, es decir las condiciones en las que se desenvuelve el ser humano, como producción, reproduc- ción, el disfrute de una vida digna, que tienden a mejorar los estilos de vida y por consecuencia tener un mejor estado de salud (3, 10,13). El presente artículo de investigación surge del interés de los integrantes del equipo hacia la enseñanza, el control y el buen manejo del autocuidado en los alumnos de nuevo ingreso de las distintas carreras de la Unidad Académica Multidisciplinaria Zona Media.

\section{MATERIAL Y MÉTODOS}

Es una investigación que se sustenta bajo el paradigma cuantitativo, corresponde a un diseño descriptivo, correlacional, de corte transversal, realizado en la Unidad Académica Multidisciplinaria Zona Media, incluyendo el total de 281 individuos que constituyó la población de alumnos de nuevo ingreso que pertenecen a las distintas carreras: Licenciatura en Enfermería, Contador Público, Ingeniero Civil, Mercadotecnia, Administración, Ingeniero en Mecatrónica e Ingeniero Agrónomo, durante el año 2012, y con una edad que varía de entre 16 a 34 años. Las encuestas realizadas fueron informadas a los alumnos y se aplicaron en las aulas de estudio de la institución de manera voluntaria durante el periodo septiembre a octubre de 2012. Identificando las variables de estudio como sexo, edad, agencia de autocuidado se aplicaron cuatro encuestas: Escala de autocuidado (ASA), Motisalud, SF-36 y APGAR Familiar. La escala ASA, desarrollada para medir el concepto central de la teoría de enfermería de déficit de autocuidado de Orem, desarrollada por Evers, se compone de 24 ítemes con un formato de respuesta de 5 alternativas tipo Likert, donde el 1 (totalmente en desacuerdo) significa el valor más bajo de capacidad de autocuidado y el 5 (totalmente de acuerdo) es el más alto (17). La escala Motisalud consta de 20 ítemes y está diseñada para medir la motivación hacia la salud, comprende seis dimensiones: Preocupación general por la salud, 
Instrumentación, Evitación del estrés, Persistencia, Orientación preventiva y búsqueda de información. La encuesta SF-36 considera datos básicos como: actividades o cosas que usted podría hacer en un día normal; problemas en su trabajo o en sus actividades cotidianas, cómo se ha sentido y cómo le ha ido durante las 4 últimas semanas. El funcionamiento familiar fue evaluado con el APGAR Familiar, el cual consta de 5 ítemes que recogen la percepción de disfunción familiar por el sujeto que contesta, mediante cinco variables relacionadas con el funcionamiento familiar. Además, este mismo instrumento fue diseñado para la aplicación de la prueba piloto que se aplicó a una muestra total de 58 alumnos. Para el procesamiento estadístico de los datos se utilizó el Statistical Package for the Social Sciences (SPSS) versión 19.0; aplicándose frecuencias, promedios, porcentajes, desviación estándar y tablas de contingencia para la estadística descriptiva; para el análisis correlacional-causal de las variables independientes y dependiente se utilizó un análisis ANOVA.

\section{RESULTADOS}

La investigación se llevó a cabo en la Unidad Académica Multidisciplinaria Zona Media, la cual se trabajó con 281 alumnos de nuevo ingreso que están dispersos en las distintas carreras que imparte la institución, como Licenciatura en Enfermería 23.8\%, Administración 16.7\%, Ingeniería Civil 18.1\%, Mercadotecnia 12.1\%, Contador Público 9.6\%, Mecatrónica 9.3\% e Ingeniería Agroindustrial 10.3\%. Los alumnos pertenecen a un rango de edad que varía de 16 a 34 años, con edad promedio de 18,5 años; el 52\% corresponde a un total de 145 alumnos de sexo femenino y el $48 \%$ se refiere a un total de 136 personas de sexo masculino. Una percepción de ingresos familiares mensuales en los estudiantes muestra un alto porcentaje de ingreso familiar mensual de $\$ 1.600$ a $\$ 2.500$ que correspon- de a un porcentaje del $29.2 \%$ y consecuente con ellos tenemos el $27 \%$ de estudiantes que percibe el mayor ingreso, más de $\$ 3.500$. El estado civil predominante corresponde a la condición de soltero, $96 \%$, y la minoría de los estudiantes en condición de separado, $1 \%$. Se destaca además la existencia de estudiantes casados con un $2 \%$ y con el estado civil de unión libre de $1 \%$. La procedencia de los estudiantes es por minoría: un $4 \%$ proviene de otra ciudad distinta a la urbana, $30 \%$ de procedencia rural y por mayoría, $66 \%$ estudiantes provenientes de zonas urbanas, lo que estaría evidenciando una mayor oportunidad de acceso a la Universidad para este grupo de jóvenes; además el servicio de salud de los estudiantes muestra un $27 \%$ que están afiliados al servició de salud IMSS por parte de sus padres o por el seguro facultativo; una minoría de $6 \%$ de estudiantes son derechohabientes del ISSSTE, 24\% afiliados al Seguro Popular y el $24 \%$ de los estudiantes no cuentan con ningún tipo de servicio de salud y asisten a clínicas particulares. Se destaca, además, un $19 \%$ de estudiantes con valor perdido a causa de no haber contestado este apartado en las encuestas (Tabla 1). El funcionamiento del APGAR Familiar consta de 5 ítemes que recogen la percepción de disfunción familiar por el sujeto que contesta, mediante cinco variables relacionadas con el funcionamiento familiar; como resultado destaca que $29 \%$ percibe algún grado de disfunción en sus familias, lo que corresponde a 82 de 281 alumnos de nuevo ingreso. Lo cual se interpreta como que el estudiante no tiene una buena relación con sus familiares por diversos factores, pudiendo tomar en cuenta la distancia, el tiempo, el estado civil de los padres o el nivel económico, por mencionar algunos (Gráfico 1). Como resultado a la encuesta SF36 , muestra que un $98 \%$ corresponde a tener un valor de escala SF-36 alto, se consideraron datos básicos del encuestado, tales como: actividades o cosas que usted podría hacer en un día normal; problemas en su trabajo o en sus actividades cotidianas; cómo se ha senti- 
Tabla 1. Distribución porcentual de resultados obtenidos de los estudiantes de nuevo ingreso. Noviembre 2012.

\begin{tabular}{llr}
\hline Característica & Indicador & $\%$ \\
\hline & Enfermería & 23.8 \\
& Administración & 16.7 \\
Carrera & Ingeniería civil & 18.1 \\
& Mercadotecnia & 12.1 \\
& Contador público & 9.6 \\
& Mecatrónica & 9.3 \\
& Agroindustrial & 10.3 \\
\hline Edad & De 16 a 34 años; promedio de 18.5 \\
\hline Sexo & Femenino & 48.4 \\
& Masculino & 51.6 \\
\hline \multirow{3}{*}{ Ingreso familiar mensual } & Menos de $\$ 1,600$ & 21.1 \\
& \$1,600 a \$2,500 & 29.2 \\
& \$2,500 a \$3,500 & 21.7 \\
\hline \multirow{2}{*}{ Estado civil } & Más de \$3,500 & 28.0 \\
& Soltero & 96.1 \\
& Casado & 1.8 \\
& Separado & 0.7 \\
Procedencia & Unión libre & 1.4 \\
\hline & Urbano & 65.8 \\
& Rural & 29.9 \\
& Foráneo & 4.3 \\
\hline \multirow{2}{*}{ Servicio de salud } & IMSS & 27 \\
& ISSSTE & 6 \\
& Seguro popular & 24 \\
& Ninguno & 24 \\
& Valor perdido & 19 \\
\hline
\end{tabular}

Fuente: Directa.

Gráfico 1. Distribución porcentual de resultados obtenidos de los estudiantes de nuevo ingreso, según APGAR familiar. Noviembre 2012.

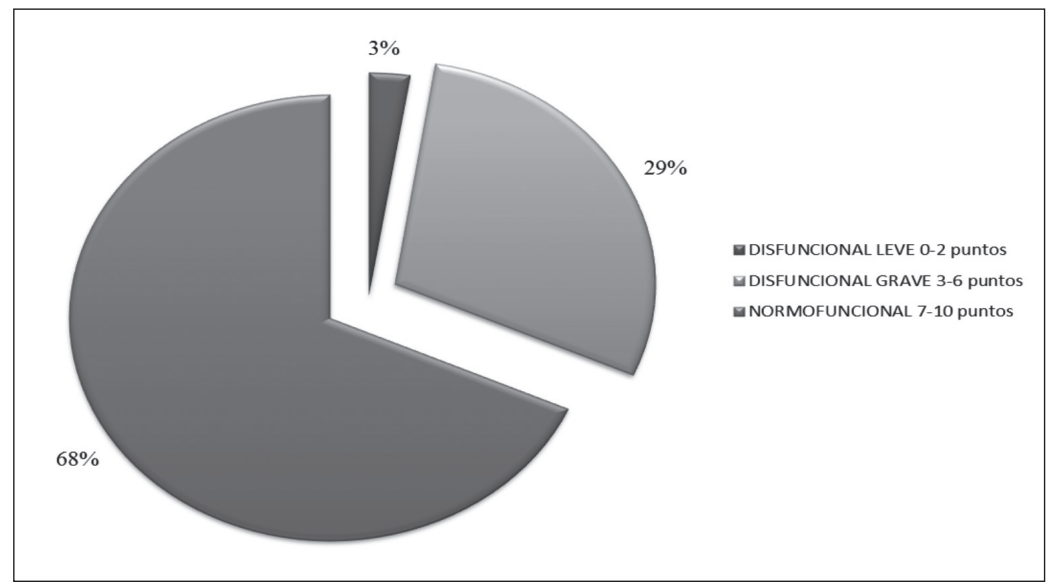

Fuente: Directa. 
do y cómo le han ido las cosas durante las 4 últimas semanas (Gráfico 2). La escala Motivación hacia la salud MOTISAL, está compuesta por 20 ítemes, de los cuales se valora de acuerdo a lo siguiente: Completamente en desacuerdo (5), Más bien en desacuerdo (4), $\mathrm{Ni}$ de acuerdo, Ni en desacuerdo (3), Más bien de acuerdo (2) y Completamente de acuerdo (1), sumando al final los puntos de cada ítem (68 puntos) y después clasificarlos en bajo de
0 a 22 puntos, mediano de 23 a 45 puntos y alto nivel de autocuidado de 46 a 68 puntos, y como resultado muestra un $96 \%$ con un alto nivel de motivación hacia la salud, y un 3\% con un mediano nivel de motivación hacia la salud y un $1 \%$ muy baja motivación. Con esto se concluye que los alumnos de nuevo ingreso de la Unidad Académica Multidisciplinaria de la Zona Media tienen alta motivación en la salud (Gráfico 3). El instrumento ASA

Gráfico 2. Distribución porcentual de resultados obtenidos según escala SF-36. Noviembre 2012.

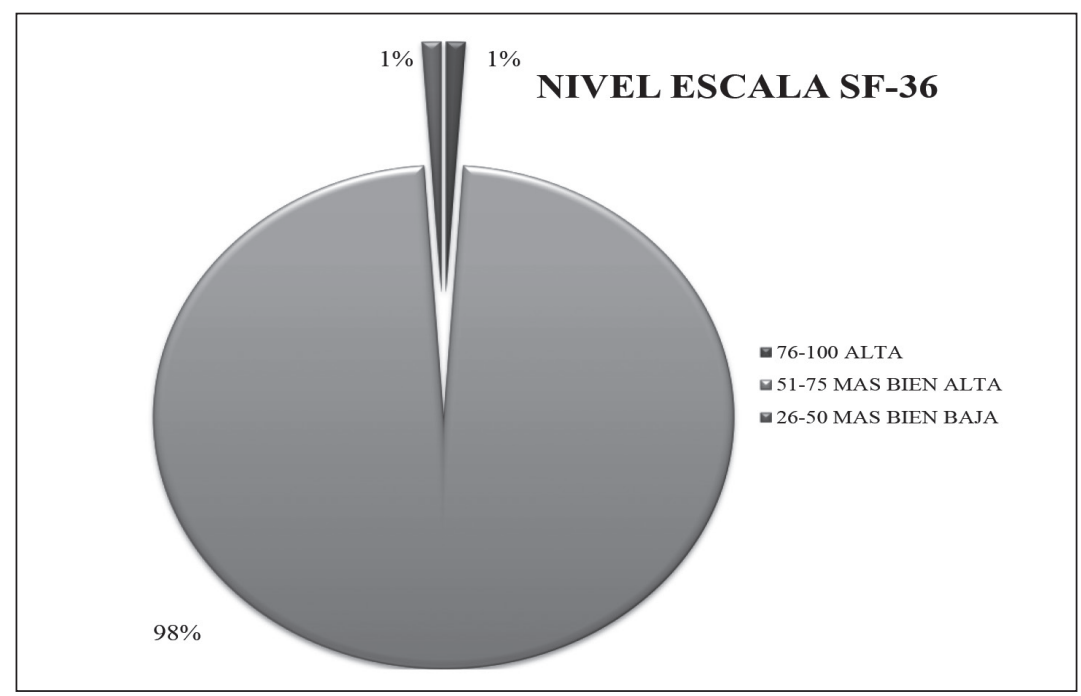

Fuente: Directa.

Gráfico 3. Nivel de motivación hacia la salud, según la escala MOTISAL.

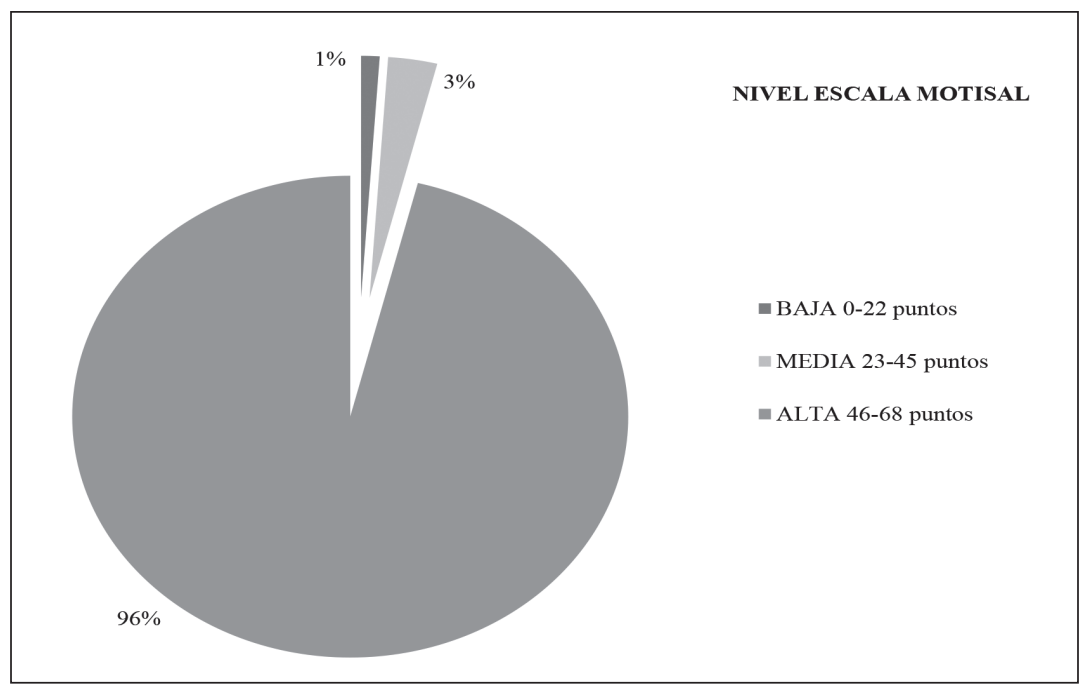

Fuente: Directa. 
está compuesto por 24 ítemes que miden el autocuidado y se evalúa de la siguiente manera: "SIEMPRE" (4), "CASI SIEMPRE"(3), "CASI NUNCA"(2) y "NUNCA"(1), sumando al final los puntos de cada ítem (96 puntos) para después clasificarlos en mediano de 49 a 72 puntos y alto nivel de autocuidado de 73 a 96 puntos y un valor "No aplica" que incluye a aquellos alumnos que dejaron sin contestar un ítem del instrumento. De los cuales resulto un $54 \%$ con mediano nivel de autocuidado, $42 \%$ con alto cuidado y $4 \%$ no aplica. Con esto se concluye que los alumnos de nuevo ingreso de la Unidad Académica Multidisciplinaria de la Zona Media tienen conocimiento de cómo cuidar su salud (Gráfico 4).

En la Tabla 2 se muestran los valores de los factores comparados con la escala de valoración de la agencia de autocuidado ASA, dichos resultados nos muestran que solamente el valor de la carrera tiene una significancia válida con un resultado de .001, esto nos da un iteres hacia cada carrera para saber cuál va a ser el método de autocuidado que mejor les convenga.

Gráfico 4. Nivel de autocuidado según la escala ASA.

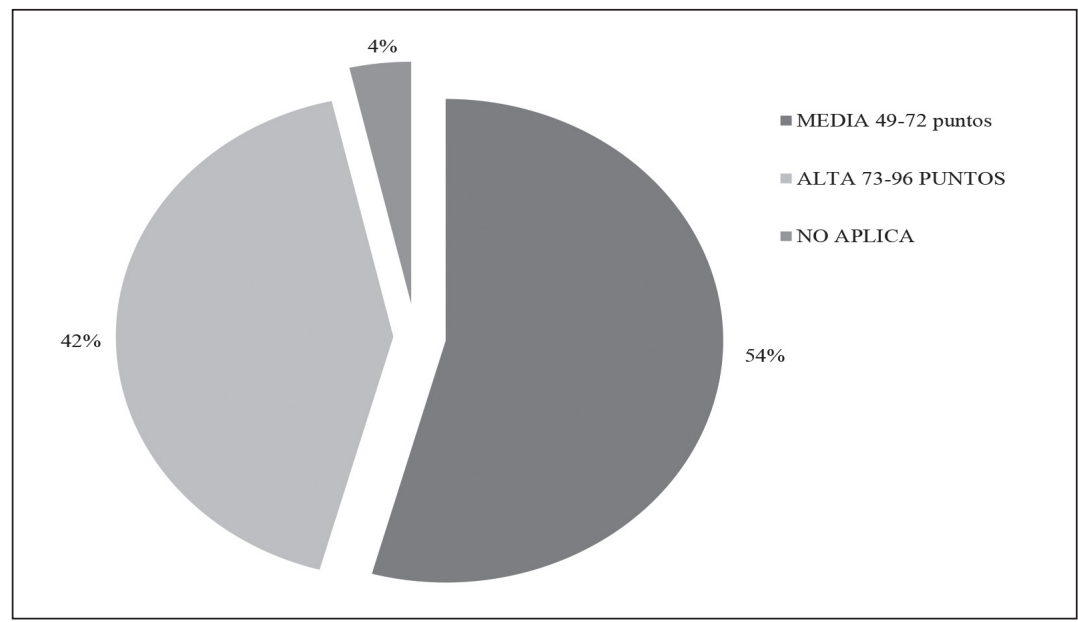

Fuente: Directa.

Tabla 2. Tabla de comparación de significancia de análisis ANOVA de la escala de autocuidado ASA con diferentes variables. Noviembre 2012. EVALUADOR 3, OBSERVACIÓN 8 y 10.

\begin{tabular}{lrcc}
\hline Variable & gl & F & Sig. \\
\hline \multirow{2}{*}{ Género } & 1 & 3.648 & 0.057 \\
& 278 & & \\
\hline \multirow{2}{*}{ Edad } & 279 & & \\
& 12 & 1.699 & 0.067 \\
\multirow{2}{*}{ Estado Civil } & 267 & & \\
& 279 & & \\
\hline \multirow{2}{*}{ Carrera } & 3 & 0.991 & 0.397 \\
& 276 & & \\
\hline
\end{tabular}




\begin{tabular}{lrrc}
\hline & 10 & 1.506 & 0.137 \\
APGAR Familiar & 269 & & \\
& 279 & & 0.756 \\
Escala SF-36 & 2 & 0.280 & \\
\hline & 276 & & 0.868 \\
Escala MOTISAL & 278 & & \\
\hline
\end{tabular}

Fuente: Directa.

\section{DISCUSIÓN Y CONCLUSIÓN}

El presente estudio tuvo como finalidad identificar la relación de las capacidades y acciones de autocuidado con los indicadores de salud: en un grupo de adultos jóvenes universitarios de las diferentes carreras de la Unidad Académica Multidisciplinaria de la Zona Media.

Este estudio permitió conocer cómo son las capacidades y acciones de autocuidado de los jóvenes universitarios, situación que muestra que a pesar de tener los conocimientos para cuidarse de sí mismos muestran un déficit de autocuidado (15). Se utilizaron 4 instrumentos en relación a las variables y acciones de autocuidado, al encontrar capacidades estadísticamente altas en los sujetos de estudio, pero disminuidas en sus acciones de autocuidado (APGAR Familiar, SF-36, Motisalud y ASA); además se adicionó 6 ítemes que son: sexo, edad, procedencia, estado civil, nivel económico y servicio de salud; éstos fueron de relevancia, en el sentido que se encontró de un total de 281 participantes, donde predomina el sexo femenino con el $52 \%$ y $48 \%$ masculino, en comparación con el artículo "Factores condicionantes básicos asociados al estado nutricional de los alumnos de nuevo ingreso en una institución de educación superior", donde se encontró que predomina el sexo femenino (58\%) de una población de 243 alumnos de nuevo ingreso en la Unidad Académica Multidisciplina- ria Zona Media, pertenecientes la mayoría a un sistema de atención en salud pública, que va a depender del estilo de vida según circunstancias socioeconómicas y de salud, se obtuvo un mayor número de mujeres en la carrera de enfermería, al igual que en nuestro estudio de comparación, siendo también como edad mínima 16 años $(17,18)$. Reyes de León de Izaguirre ${ }^{2}$ refiere que la población no tiene un buen aporte económico, al igual que nuestro estudio de comparación, que muestra un bajo ingreso para llevar a cabo una buena alimentación, tener autocontrol y así mantener la vida, salud y bienestar favorables de sí mismo (19).

El instrumento "APGAR Familiar" está compuesto por 5 ítemes, los cuales recogen información acerca de la función familiar, clasifica en un rango de funcionales de 7 a 10 puntos, moderadamente disfuncionales aquéllos con puntuación entre 4 a 6 puntos y gravemente disfuncionales los que tienen de 0 a 3 puntos, entre los cuales destacan las familias funcionales con un porcentaje de $68 \%$, éste tiene un impacto favorecedor ya que los estudiantes reciben apoyo de la familia cuando algo les molesta o perjudica, comparten los problemas, se encuentran satisfechos con sus familias y con sí mismos para emprender nuevas metas, apoyos en sus decisiones, prácticas cotidianas, compartir tiempo y es-

\footnotetext{
${ }^{2}$ Reyes de León de Izaguirre MR. Capacitación de la familia para el Autocuidado. [Tesis de licenciatura]. Guatemala: Universidad Mariano Gálvez de Guatemala; 2004.
} 
pacio (20). El apartado de la encuesta "SF36 " está compuesto por nueve ítemes en los cuales evalúa la salud de los estudiantes universitarios en relación con la actividad física y la presencia de estados depresivos, además nos informa de cómo el estudiante se ha sentido durante el último mes con respecto a su motivación en la vida. Los datos revelan que el $98 \%$ es más bien alta en comparación del $1 \%$ que es más bien baja y un $1 \%$ alta. Como resultado, el realizar actividad física favorece al estado de salud en el alumno de nuevo ingreso en la Unidad Académica Multidisciplinaria Zona Media; son funciones que ayudan al buen accionar del sistema circulatorio y mejora el metabolismo, esto trae como consecuencia una menor tasa de estados depresivos y por lo tanto se tiene mejor motivación, mejores hábitos y una marcada disminución en los suicidios. Datos similares a los que presentan Choque y Escalante ${ }^{3}$, en donde mencionan que es una distracción buena para los estudiantes en edades tempranas, es una prevención en el consumo de sustancias como el alcohol, tabaco y drogas, y así con la realización de ejercicio incrementar su autocuidado (21-23). La escala Motisalud consta de 20 ítemes diseñados para medir la motivación hacia la salud y consta de seis dimensiones: Preocupación general por la salud, instrumentación, evitación del estrés, persistencia, orientación preventiva y búsqueda de información. Como respuesta, el $96 \%$ de la población estudiantil está satisfecho con la forma en la que han manejado su salud hasta ahora, por lo cual se interpreta que los jóvenes de nuevo ingreso incluidos a esta investigación resultaron con alta motivación hacia el mejoramiento de su salud en su vida diaria, mantienen cuidados y como consecuencia están saludables $(24,25)$. La escala "ASA" fue desarrollada para medir el

${ }^{3}$ Choque CD, Escalante MP. Conductas de Autocuidado de la Salud en Estudiantes de 18 a 25 años que Asisten al Examen Preventivo de la Salud en la UNC. Proyecto de Investigación. [Tesis]. Córdoba: Facultad de Ciencias Médicas; 2010. concepto central de la teoría de enfermería de déficit de autocuidado de Orem, se compone de 24 ítems con un formato de respuesta de 5 alternativas tipo Likert y como resultado el $54 \%$ de la población estudiantil universitaria muestra un rango medio y como consecuencia arroja que los estudiantes de nuevo ingreso tienen conocimiento sobre la forma en cómo afrontar un problema de salud y estar con un potencial humano saludable, aunque no se asegura que los alumnos de la unidad lo pongan en práctica en su vida diaria como se describe por Rebolledo Oyarzo ${ }^{4}$, Martínez Hernández y Sáez Muñoz ${ }^{5}$ con resultados similares (26). El artículo de comparación realizado en el 2011 no menciona el nombre de los instrumentos que se utilizaron para la elaboración del proyecto, solo describe que está compuesto por 19 ítemes.

La agencia de autocuidado puede verse afectada en su desarrollo y operatividad por una serie de factores que fueron analizados en este estudio junto a otras variables consideradas. Los factores analizados que se asociarían con la agencia de autocuidado de los estudiantes de nuevo ingreso (Generación 2012-2016) de la Universidad Autónoma de San Luis Potosí, Unidad Académica Multidisciplinaria de la Zona Media. La educación sobre autocuidado en los universitarios debe ser un proceso permanente. Estudios como éste ofrecen la oportunidad para identificar y cambiar hábitos que signifiquen mejorar la calidad del conocimiento y del autocuidado a corto y mediano plazo.

${ }^{4}$ Rebolledo Oyarzo DP. Autocuidado de los estudiantes de la carrera de Enfermería de la Universidad del Bío-Bío: un análisis según el Modelo de Autocuidado de Dorothea Orem. [Tesis de licenciatura]. Chile: Universidad Austral de Chile; 2010.

${ }^{5}$ Martínez Hernández D, Sáez Muñoz P. Conductas de autocuidado en estudiantes universitarios residentes del complejo de hogares Huachocopihue de la UACH, 2006. [Tesis de licenciatura]. Chile: Universidad Austral de Chile, 2007. 


\section{REFERENCIAS}

1. De la Fuente V, Omaña V, SooRan Lee R, Alavez B, Peña H, Sierra E. Conocimiento $\mathrm{y}$ acciones de los adolescentes sobre el autocuidado de su salud. Rev CONAMED. 2011; 16(1): 29-33.

2. Durá M, Pacheco J. Estilos de vida y conductas de riesgo de los adolescentes universitarios. Reduca. 2010; 2(1): 680-695.

3. Escobar M, Franco Z, Duque J. El autocuidado: un compromiso de la formación integral en educación superior. Hacia promoc. Salud. 2011; 16(2): 132-146.

4. Lema L, Salazar I, Varela M, Tamayo J, Rubio A, Botero A. Comportamiento y salud de los jóvenes universitarios: satisfacción con el estilo de vida. Pensamiento psicológico. 2009; 5(12): 71-88.

5. Báez F, Zenteno M, Flores M, Rugerio M. Capacidades, acciones de autocuidado e indicadores de salud en adultos jóvenes universitarios de enfermería. Revista Cultura del Cuidado. 2012; 9(2):10-21.

6. Pereda M. Explorando la teoría general de enfermería de Orem. UNAM. EnfNeurol (Mex). 2011; 10(3):163-167.

7. Gómez O, Sesma S, Becerril V, Knaul F, Arreola H, Frenk J. Sistema de salud de México. Salud PublicaMex. 2011; 53(2): 220-232.

8. Ibarra Mendívil JL., Gaytán Fox G., Salazar Granillo CM., Rodríguez Márquez AL. El autocuidado en los adolescentes sonorenses asignatura estatal. Programa de estudio 2011. $1^{\text {a }}$ ed. Sonora: Secretaría de Educación y Cultura del estado de Sonora; 2013.

9. Espinoza K, Jofré V. Sobrecarga, apoyo social y autocuidado en cuidadores informales. Cienc. enferm. 2012; XVIII(2): 23-30.

10. Espinoza L, Rodríguez F, Gálvez J, MacMillan N. Hábitos de alimentación y actividad física en estudiantes universitarios. RevChilNutr. 2011; 38(4): 458-465.
11. Sánchez C, Pillon S. Tabaquismo entre universitarios: caracterización del uso en la visión de los estudiantes. Rev Lat Am Enfermagem. 2011; 19: 730-737.

12. Cabrolier E, Sandoval Y. Autocuidado en adolescentes portadores de cáncer. Horiz-Enferm. 2011; 22(1): 73-81.

13. Taylor S. Teoría del déficit de autocuidado. En: Marriner A, Raile M. Modelos y teorías en enfermería. 6a ed. España: Elsevier; 2007. p. 267-295.

14. Stephen J. Cavanagh. Modelo de Orem. Aplicación práctica. Ediciones Científicas y Técnicas S.A. Barcelona: Editorial MASSON - SALVAT Enfermería, 1993.

15. Orem D, Taylor S, McLaughlin K. Nursing Concepts of Practice. 6 edition. USA: Mosby; 2001; 114-542.

16. Tobón O. El autocuidado una habilidad para vivir. Revista Hacia la Promoción de la Salud [Internet]. 2003 Nov [citado 10 enero 2013]. Disponible en: http://promocionsalud.ucaldas.edu.co/downloads/ Revista\%208_5.pdf

17. Manrique-Abril F, Fernández A, Velandia A. Análisis factorial de la Escala Valoración de Agencia de Autocuidado (ASA) en Colombia. Aquichan. 2009; 9(3): 222235.

18. Bastidas C. Asociación entre la capacidad de la agencia de autocuidado y la adherencia a tratamientos farmacológicos y no farmacológicos en personas con alguna condición de enfermedad coronaria. av.enferm. 2007; XXV(2):65-75.

19. Ibarra M, Méndez M, Muñoz T, Rocha M. Factores condicionantes básicos asociados al estado nutricional de los alumnos de nuevo ingreso en una institución de educación superior. Rev Paraninfo Digital [Internet] 2013 [citado 27 septiembre 2013 ]; (17). Disponible en: http://indexf. publicaciones.saludcastillayleon.es/para/ n17/pdf/pdf.php?p=078

20. Álvarez G, Martínez AM. Capacidades de autocuidado para la prevención del VIH en adolescentes. [Tesis de maestría]. [Mi- 
natitlán (MX)]: Universidad Veracruzana; 2011.

21. Hernández L, Zequeira D, Miranda A. La percepción del cuidado en profesionales de enfermería. Rev Cubana Enferm [Internet]. 2010 [citado 27 septiembre 2013]; 26(1):30-41. Disponible en: http:// www.bvs.sld.cu/revistas/enf/vol26_1_10/ enf070110.pdf

22. Desarrollo Programático y Fortalecimiento Institucional, Secretaría Ejecutiva del Sistema de Protección Social. Ministerio de Planificación (Gobierno de Chile). Guía para la Reflexividad y el Autocuidado Dirigido a Profesionales y Educadores de Equipos Psicosociales de los Programas del Sistema de Protección Social Chile Solidario [Internet]. Santiago: Chile Solidario, PROTEGE Red de protección social (CL); [citado 27 septiembre 2013]. 106 p. disponible en: http://www. chilesolidario.gob.cl/administrador/arc doc/5427682154ba8c2683ac7e.pdf
23. Velandia A, Rivera L. Confiabilidad de la escala "Apreciación de la agencia de autocuidado" (ASA) segunda versión en español, adaptada para la población colombiana. av.enferm. 2009; XXVII(1): 38-47.

24. Buitrago L, Molina N. Características del fenómeno de las drogas en estudiantes de la Universidad Libre Seccional Pereira. Revista Cultura del Cuidado. 2011; 8(2): 28-36.

25. Navarro Peña Y, Castro Salas M. Modelo de Dorothea Orem aplicado a un grupo comunitario a través del proceso de enfermería. Enfermería global. [Internet] 2010 [citado 27 de septiembre de 2013]; (19). Disponible en: www.um.es/englobal/

26. González B, Lupón J, Parajón T, Urrutia A, Herreros J, Valle V. Aplicación de la escala europea de autocuidado en insuficiencia cardíaca (EHFScBS) en una unidad de insuficiencia cardíaca en España. RevEspCardiol. 2006; 59(2):166-170. 